\title{
EL LENGUAJE Y LA COMPRENSIÓN DEL OTRO EN \\ LA HERMENÉUTICA DE GADAMER
}

\author{
José Luis Obregón Cabrera* \\ Pontificia Universidad Católica del Perú \\ jobregon@pucp.edu.pe
}

Fecha de recepción: agosto de 2015 Fecha de aceptación: octubre de 2015

RESUMEN: En el presente artículo se revisarán los argumentos que sostienen la tesis hermenéutica de la ontología del lenguaje que presenta Gadamer en su obra Verdady método, analizando la relación entre conceptos de comprensión,

* José Luis Obregón Cabrera es magíster y licenciado en Filosofía por la Pontificia Universidad Católica del Perú. Actualmente, se encuentra realizando, en la misma casa de estudios, su tesis doctoral en Filosofía sobre la aproximación hermenéutica al diálogo interreligioso. Asimismo, se desempeña como docente de los cursos de Filosofía, y Ciencia y Filosofía en la Facultad de Estudios Generales Ciencias de la Pontificia Universidad Católica del Perú; así como del curso de Ética y ciudadanía, en la Universidad Peruana de Ciencias Aplicadas; y del curso de Filosofía de la religión en el ISET Juan XXIII. Ha publicado "Filosofía práctica, modernidad y comprensión" (2013) en la revista Pastores del Nuevo Milenio, Año 13, N. 24, 2013, y "La hermenéutica como filosofía práctica: los aportes éticos de la experiencia hermenéutica y el lenguaje" (2010) en http://red.pucp.edu.pe/cipher/docs/jose_luis_obregon.pdf. 
lenguaje y diálogo. Con ese objetivo, se examinará, en primer lugar, el término lingüisticidad para destacar la naturaleza lingüístico-ontológica de toda comprensión. Posteriormente, se observará una tesis fundamental en la hermenéutica filosófica que consiste en entender el lenguaje como acepción del mundo, la cual se opone a las tesis reduccionistas del lenguaje como simple instrumento. Finalmente, será menester mostrar la relevancia de esta concepción hermenéutica del lenguaje, en tanto condición de posibilidad de la comprensión del otro y la preeminencia de la dimensión práctica que involucra dicha concepción.

Palabras clave: Hermenéutica, Gadamer, lenguaje, comprensión, diálogo.

\section{LANGUAGE AND UNDERSTANDING OTHERS IN GADAMER'S HERMENEUTICS}

Aвstract: In this article we will review the arguments that support the hermeneutic thesis of ontology of language that Gadamer presents in his Truth and Method, analyzing the relationship among concepts of comprehension, language and dialogue. To this end, firstly we will review the term linguisticality to highlight the linguistic-ontological nature of comprehension. Later, we will examine a fundamental thesis on the philosophical hermeneutics is to understand the language as meaning of the world, which is opposed to the reductionist thesis of language as a mere instrument. Finally, we are interested in displaying the relevance of this hermeneutic conception of language as condition for understanding others and the preeminence of the practical dimension involving this concept. KeYwords: Hermeneutics, Gadamer, language, understanding, dialogue. 


\section{EL LENGUAJE Y LA COMPRENSIÓN DEL OTRO EN \\ LA HERMENÉUTICA DE GADAMER}

\section{Introducción}

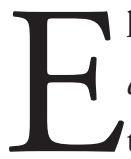

1 tema principal de la hermenéutica remite al fenómeno de la comprensión en general. En efecto, la hermenéutica en un sentido tradicional se entiende como el arte de interpretar textos. Sin embargo, la hermenéutica filosófica de Gadamer va más allá, mostrando que el hombre es un ser comprensivo, es decir, que la comprensión lo constituye en un sentido ontológico. Por eso, Gadamer, en la tercera sección de Verdad y método, planteará una ontología del lenguaje, con la finalidad de mostrar que toda comprensión tenga lugar a nivel del lenguaje.

De acuerdo con dichas bases, en este artículo se revisarán los argumentos que sostienen la tesis hermenéutica de la ontología del lenguaje, analizando la relación entre conceptos del lenguaje, el diálogo y la praxis. Con ese objetivo, se analizará, en primer lugar, el término lingüisticidad para destacar la naturaleza lingüístico-ontológica de toda comprensión. Posteriormente, se tratará una tesis hermenéutica central que consiste en entender el lenguaje como acepción del mundo. Asimismo, esta tesis insistirá en el rechazo al instrumentalismo del lenguaje, con el fin de hacer ver la dimensión práctico-política que involucra dicha concepción.

\section{El Lenguaje como Diálogo}

El tema del lenguaje en la hermenéutica gadameriana está muy emparentado con la definición clásica de Aristóteles del hombre como un ser de lenguaje: "Sólo los seres humanos poseen (...) el logos que los capacita para informarse mutuamente sobre lo que es útil y lo que es dañino, y también lo que es justo y lo que es injusto" (Aristóteles en Gadamer, 1992, p. 145). El hombre, esencialmente, es un ser de lenguaje, un ser de 


\section{JOSÉ LUIS OBREGÓN CABRERA}

logos de una capacidad propiamente humana que le permite comunicarse con otros y determinar aquello que considera conveniente. Al respecto, Gadamer destacará dos aspectos relevantes en esta definición aristotélica para su propia concepción hermenéutica del lenguaje:

Se establece, pues, aquí como nota característica del hombre una superioridad sobre lo actual, un sentido de futuro. (...) Puede comunicar todo lo que piensa; y lo que es más, gracias a esa capacidad de comunicarse las personas pueden pensar lo común, tener conceptos comunes, sobre todo aquellos conceptos que posibilitan la convivencia de los hombres sin asesinatos ni homicidios. (1992, p. 145)

Gadamer destaca que, mediante el lenguaje, el hombre puede referirse a cosas futuras, puede proyectar su propia vida, haciendo presente algo que aún no lo es. Pero, además, deja entrever el lenguaje como un medio de praxis vital. Ello debido a que, mediante el lenguaje, el hombre puede pensar en comunidad, es decir, puede compartir con otros hombres conceptos e ideas que hagan posible la vida en común.

Ahora bien, esta concepción del lenguaje como medium de la propia praxis vital está vinculada a la concepción heideggeriana del lenguaje como acontecimiento. Para Gadamer, esta expresión de resonancias heideggerianas quiere dar a entender que en el lenguaje acontece algo que no depende necesariamente de quienes participan en él: "el lenguaje que discurre en la conversación lleva consigo su propia verdad, esto es, desvela y deja aparecer algo que desde ese momento es" (1977, p. 461). El lenguaje es acontecimiento no porque sea algo puramente manipulable. Es acontecimiento porque ocurre algo que los participantes no son capaces 


\section{EL LENGUAJE Y LA COMPRENSIÓN DEL OTRO EN \\ LA HERMENÉUTICA DE GADAMER}

de dominar completamente. Esta concepción tendrá implicancias en el concepto gadameriano de lenguaje que se define más específicamente como diálogo:

La hermenéutica afirma que el lenguaje pertenece al diálogo (Gespräch); es decir, el lenguaje es lo que es si porta tentativas de entendimiento (verständingungsversuche), si conduce al intercambio de comunicación, a discutir el pro y el contra. El lenguaje no es proposición y juicio, sino que únicamente es si es respuesta y pregunta. (Gadamer, 1993, p. 119)

Aquí Gadamer aludirá a un tema recurrente en su filosofía: el lenguaje no es mera proposición, pues no es un simple instrumento. La hermenéutica rechaza las posiciones instrumentalistas del lenguaje, donde se le concibe meramente como un conjunto de reglas gramaticales disponibles para los hombres. Por el contrario, Gadamer sostendrá que el lenguaje es diálogo vivo, porque se realiza en la conversación como intercambio de preguntas y respuestas.

Cuando el filósofo habla del diálogo vivo subraya el hecho de que es acontecimiento, porque no es ni predecible ni controlable por los interlocutores. A su vez, el rumbo del diálogo es incierto. En efecto, los interlocutores no saben a dónde serán conducidos por el diálogo, puesto que ellos están abiertos a encontrar la verdad como un sentido compartido. El diálogo es un proceso a través del cual los hablantes llegan a entenderse mutuamente mediante la escucha del otro: "Forma parte de toda verdadera conversación el entender realmente al otro, dejar valer sus puntos de vista" (Gadamer, 1977, p. 463). 
Igualmente, Gadamer no olvidará la condición que hace posible el diálogo como tal: el diálogo demanda que el interlocutor esté dispuesto a no tener la razón e incluso a fortalecer la opinión del otro. En ese sentido, el diálogo transforma los puntos de vista, modifica las diferentes posiciones iniciales de cada interlocutor: "La verdadera realidad de la comunicación humana consiste en que el diálogo no impone la opinión de uno contra la del otro ni agrega la opinión de uno a la de otro a modo de suma. El diálogo transforma una y otra” (1992, p. 184).

Desde esta perspectiva del lenguaje como diálogo vivo, debe entenderse la afirmación gadameriana del lenguaje "como un estar en camino a lo común de unos con otros" (Gadamer, 1993, p. 119). Así, el lenguaje lleva consigo la posibilidad del entendimiento mutuo, pues por medio del lenguaje los hombres pueden ponerse de acuerdo y compartir algo común.

Por esa razón, Gadamer también señalará que una conversación es semejante a una traducción: así como en una conversación los dialogantes están dispuestos a hacer valer la opinión del otro; así también el traductor tiene que hacer valer lo extraño y desfavorable que el texto puede manifestar, porque se trata de llegar a un acuerdo entre las partes (Gadamer, 1977). En ese sentido, una traducción es siempre una interpretación. Ahora bien, lo central en ello es que, se trate o no de una traducción, todo ponerse de acuerdo presupone un proceso lingüístico, ya que todo entendimiento se realiza en el lenguaje: "el lenguaje es el medio universal en el que se realiza la comprensión misma. La forma de realización de la comprensión es la interpretación” (Gadamer, 1977, p. 467).

Esta es una tesis primordial sobre el lenguaje que desarrollará Gadamer en Verdady método, la comprensión es un hecho lingüístico, incluso cuando se trata de comprender algo extralingüístico: "no sólo el proceso 


\section{EL LENGUAJE Y LA COMPRENSIÓN DEL OTRO EN \\ LA HERMENÉUTICA DE GADAMER}

interhumano de entendimiento, sino el proceso mismo de comprensión es un hecho lingüístico incluso cuando se dirige a algo extralingüístico o escuchamos la voz apagada de la letra escrita” (1992, p. 181). Ahora bien, cuando Gadamer (1992) se refiere al lenguaje como 'medio' universal utiliza un nuevo término: lingüisticidad. Por ello, con el fin de explicar por qué el fenómeno de la comprensión en general es un fenómeno lingüístico, se describirá el significado de la lingüisticidad.

\section{La Lingüisticidad como Fundamento del Lenguaje}

La lingüisticidad de la comprensión se concibe claramente en el fenómeno de la traducción, porque la traducción de un texto requiere que el texto sea de naturaleza lingüística. Ahora bien, esto significa que la interpretación lingüística representa a la interpretación en general. En efecto, siempre que la persona comprende, tiene lugar una interpretación lingüística, incluso cuando se trata de comprender un cuadro o una obra musical. Aunque estas formas de interpretación no son en sí mismas enunciadas, sí presuponen la lingüisticidad, ya que se presupone el poder comprenderlas.

Aquello se verifica en la experiencia de la comprensión de una obra de arte. Cuando se contempla una obra de arte, se lleva a cabo un esfuerzo de comprensión de la obra, aunque parezca imposible enunciarla con palabras. A pesar de no poder dar cuenta de su significado, sin embargo, persiste la intención de comprenderla y de buscar las expresiones para poder aprehender el sentido del objeto.

Esto significa que la lingüisticidad es el fundamento del lenguaje, pues todo objeto de la comprensión (sea literario o no) supone una "estructura lingüística de sentido" (Grondin, 2003, p. 195). De ahí que las obras de 
arte pueden ser comprendidas, a pesar de que el lenguaje mismo parezca insuficiente para expresar cabalmente lo que las obras de arte quieren decir. Por ese motivo, Gadamer no habla de lenguaje sino de lingüisticidad o de "carácter lingüístico". El término en la lengua alemana es Sprachlichkeit, y se traduce como 'carácter lingüístico’ o 'lingüisticidad' (Gadamer, 1998). Con ello se requiere subrayar que no necesariamente las palabras determinan la comprensión de la cosa; pues el acto comprensivo incluye, además de las palabras, los gestos, las miradas o expresiones de otra índole.

La lingüisticidad excede al lenguaje en cuanto refiere a las personas a otras formas de comunicación, por este motivo Gadamer afirmará: "Desde el momento en que las personas deben convivir tienen que poner en práctica la fantasía, imaginación, sensibilidad, simpatía, el tacto” (1998, p. 233). Ello se muestra especialmente en aquellas personas que no poseen habla. Así, por ejemplo, Gadamer mencionará que, a pesar de que el recién nacido aún no posea lenguaje, sin embargo, la lingüisticidad hará posible el entendimiento sin palabras entre el bebé y su madre.

Ahora bien, lo que definirá específicamente a la lingüisticidad es la imbricación entre lenguaje y pensamiento. Por eso se propondrá Gadamer: "a pesar de toda la diversidad de las maneras de hablar intentaremos retener la unidad indisoluble de pensamiento y lenguaje tal como la encontramos en el fenómeno hermenéutico, como unidad de comprensión e interpretación" (1977, p. 483). De igual forma, debe tenerse en cuenta que, en Verdad y método, Gadamer se propone examinar en qué consiste esa unidad de pensar y hablar en medio de una diversidad de maneras de hablar. En tal sentido, reconocerá que la pregunta hermenéutica parte de un presupuesto que comparte con la ciencia del lenguaje, a saber, la unidad interna entre lenguaje y pensamiento. 


\section{EL LENGUAJE Y LA COMPRENSIÓN DEL OTRO EN \\ LA HERMENÉUTICA DE GADAMER}

No obstante, como observará Jorge Reyes, dicha imbricación de lenguaje y pensamiento "se despliega sobre el trasfondo de una forma de vida intersubjetivamente compartida (...)" (2006, p. 170). Esto quiere decir que la lingüisticidad vendría a ser un espacio de sentido no dable a la intuición sensible, sino el ámbito pre-reflexivo donde adquieren significado todas las intuiciones. La lingüisticidad vendría a ser, entonces, la condición de posibilidad del acto comprensivo, en tanto espacio de sentido que trasciende a los lenguajes y que abarca a todos los seres humanos.

De acuerdo con esta interpretación, la hermenéutica filosófica pone así énfasis en ese presupuesto universal: la pertenencia pre-reflexiva a un espacio de sentido, el cual no aparece como objeto, pero condiciona toda manifestación de objetos (Reyes, 2006). En un sentido análogo, Richard Palmer sostendrá que la lingüisticidad corresponde al ámbito que condiciona toda comprensión: "La lingüisticidad aporta el terreno común en el que y sobre el que aquellos se pueden encontrar. El lenguaje es el medio en el que la tradición se oculta y se transmite" (2002, p. 257). Por tal motivo, el presupuesto universal de la lingüisticidad abarcará toda comprensión, incluso la comprensión de las ciencias naturales.

Entendida de esa manera, la lingüisticidad vendrá a ser ese fundamento común del que participan los seres humanos, y que define al hombre como tal. De ahí que Gadamer sostenga que la universalidad del lenguaje se mantenga a la altura de la razón. Servirá como apoyo, entonces, la siguiente afirmación del filósofo alemán:

El lenguaje rebasa cualquier argumentación contra su competencia. Su universalidad se mantiene a la altura de la razón. La conciencia hermenéutica se limita aquí a participar 
en lo que hace la relación general de lenguaje y razón. (...) El lenguaje es el lenguaje de la razón misma. (1977, p. 482)

El lenguaje es el lenguaje de la razón, porque la universalidad del lenguaje estará presente en todo aquello que sea racional, es decir, en todo aquello que es susceptible de un acuerdo compartido. Una muestra de ese acuerdo vendría a ser la experiencia musical. Incluso allí, donde aparentemente no hay palabras, las personas participan de la lingüisticidad, ya que comprenden y comparten una música en común, formando al mismo tiempo una comunidad en la misma experiencia (Gadamer, 2002).

En otras palabras, que el fenómeno de la comprensión sea un fenómeno lingüístico significa que la lingüisticidad fundamenta y construye ese esfuerzo de ponerse de acuerdo, una especie de acuerdo tácito en donde adquiere sentido la comunicación con el otro. Por eso, Gadamer afirmará que "el entendimiento es más originario que el malentendido" (1992, p. 184). Esto quiere decir que conversar o comprender al otro equivale al restablecimiento de ese entendimiento originario que constituye el mundo en el cual vivimos. De ahí que el principio supremo de la hermenéutica filosófica, es decir, el presupuesto que está a la base de cualquier forma de comprensión será:

[El deseo de] reunirnos con el otro, obtener su aprobación o, por lo menos, que se retomara lo dicho, aun cuando fuese a modo de réplica u oposición. En una palabra: queremos encontrar un lenguaje común. A esto se le llama conversación. (Gadamer, 1998, p. 227) 


\section{EL LENGUAJE Y LA COMPRENSIÓN DEL OTRO EN \\ LA HERMENÉUTICA DE GADAMER}

Como puede verse, la prioridad del diálogo radica en que hace posible la solidaridad y el encuentro con el otro en un lenguaje común. Pero esto supone que la verdadera comunicación tiene lugar cuando el diálogo no pretende imponer una opinión. Por el contrario, pretende transformar las opiniones y orientarlas hacia un lenguaje común, una interpretación común del mundo que posibilite la solidaridad moral y social (Gadamer, 1992).

\section{El Lenguaje como Acepción del Mundo}

Ahora bien, esa concepción instrumentalista del lenguaje ha perdurado especialmente en el ámbito de las ciencias naturales. En esa línea, Gadamer planteará una interesante interpretación del relato de la torre de Babel, donde destaca la pretensión humana de encontrar el lenguaje único, un lenguaje común a todos, con el fin de dominar la naturaleza: "la unidad y la solidaridad de un lenguaje común, que encarna energías indomables de la voluntad y una confianza sin límites en la propia vocación para la dominación" (1993, p. 112).

Es particularmente apreciable este relato bíblico, porque Gadamer piensa que la imagen de la torre de Babel representa a su modo el problema griego de la unidad y la pluralidad. Ese lenguaje único al que aspira nuestra babélica sociedad moderna viene a ser la matemática: "Nuestra situación mundial es, por consiguiente, ésta: el conjunto de fórmulas dominantes ha posibilitado una técnica y simbología matemáticas de una grandiosa perfección” (1993, p. 116). No puede cerrarse los ojos ante lo que ocurre en este mundo. Es indudable que los grandes adelantes científicos y tecnológicos, indispensables para la supervivencia de los hombres, han sido posibles gracias al desarrollo de las matemáticas. 
Sin embargo, Gadamer no confiará del todo en que (siguiendo la metáfora de la torre de Babel) el establecimiento de ese lenguaje único vaya a poner fin a los problemas que atraviesa la humanidad. Las matemáticas son limitadas para enfrentar las dificultades humanas sobre la convivencia entre los pueblos y la ecología, porque no son un lenguaje como tal, son un sistema de comunicación que está al nivel de una simple herramienta: "La matemática con la que el físico obtiene y formula sus conocimientos no es un lenguaje propio, sino que pertenece al instrumental lingüístico plural con el que expresa lo que quiere decir" (Gadamer, 1992, p. 188). Por ello, los problemas humanos requieren de otra instancia que haga viable soluciones razonables: este es el lugar del lenguaje.

Frente a esta problemática, la hermenéutica pone especial atención a la teoría humboldtiana sobre el lenguaje, que parte del supuesto de que la individualidad constituye un camino para comprender la totalidad de la constitución lingüística humana: "Su punto de partida es que las lenguas son productos de la 'fuerza del espíritu' humano" (Gadamer, 1977, p. 527). Humboldt piensa que en todo lenguaje actúa una fuerza lingüística originaria que aspira a alcanzar la perfección. Sin embargo, como indica Cecilia Monteagudo, la teoría de Humboldt padece de una ambivalencia: por un lado, defiende la pluralidad de las lenguas, y por otro, se mantiene en la exigencia de encontrar una instancia universal que supere la pluralidad (Monteagudo, 2009a).

Aunque Gadamer discrepará con algunos aspectos de la teoría humboldtiana, sin embargo, reconocerá que con su concepción del lenguaje como acepción del mundo el problema del lenguaje gana una dimensión fundamental. Esta tesis va contra la concepción dogmática de los gramáticos, en cuanto señala que la esencia del lenguaje se encuentra en la realización del mismo hablar. De ello, deducirá Gadamer que el hombre tiene un mundo 


\section{EL LENGUAJE Y LA COMPRENSIÓN DEL OTRO EN \\ LA HERMENÉUTICA DE GADAMER}

en la medida en que se lo representa de forma lingüística: "Para el hombre el mundo está ahí como mundo, en una forma bajo la cual no tiene existencia para ningún otro ser vivo puesto en él. Y esta existencia del mundo está constituida lingüísticamente" (1977, p. 531).

Las lenguas son acepciones del mundo porque mediante el lenguaje el individuo se sumerge en una relación determinada con el mundo y con determinados usos y costumbres. El análisis etimológico de la palabra alemana 'mundo' (welt) como proveniente de 'hombre' (wer) revelará para Gadamer algo similar a lo que Humboldt había propuesto: “mundo es mundo humano, mundo del hombre (Menschenwelt). Este es el significado originario en las lenguas germánicas e indogermánicas" (1993, p. 120). Esto implica la existencia de una relación intrínseca entre el lenguaje y el mundo, por lo cual el lenguaje no posee una existencia autónoma.

Más aún, el lenguaje tiene existencia en cuanto en él el mundo es representado: "La humanidad originaria del lenguaje significa, pues, al mismo tiempo la lingüisticidad originaria del ser-en-el-mundo del hombre" (Gadamer, 1977, p. 531). Esto significa que el hombre se encuentra dentro de esta lingüisticidad originaria, o mejor dicho, no puede tomar distancia del lenguaje, incluso cuando se esfuerce por entender lo que es el lenguaje mismo. A continuación, servirá de apoyo la siguiente aseveración de Gadamer:

La esencia del lenguaje implica una inconsciencia realmente abismal del mismo. (...) Sólo podemos pensar dentro del lenguaje, y esta inserción de nuestro pensamiento en el lenguaje es el enigma más profundo que el lenguaje propone al pensamiento. (1992, p. 147) 
JOSÉ LUIS OBREGÓN CABRERA

Esta cita expresa con toda contundencia que el lenguaje no es un medio ni un instrumento que esté al servicio de alguna ciencia, o que pueda manipularse al propio antojo. Por el contrario, son las personas en el lenguaje. Como anotará el mismo Gadamer: "Aprender a hablar no significa utilizar un instrumento ya existente para clasificar ese mundo familiar y conocido, sino que significa la adquisición de la familiaridad y conocimiento del mundo mismo tal como nos sale al encuentro" (1992, p. 148).

Por ese motivo, Gadamer también definirá el lenguaje como autoolvido, porque "la estructura, gramática, sintaxis, etc., todo lo que tematiza la ciencia, queda inconsciente para el lenguaje vivo. (...) El lenguaje real y efectivo desaparece detrás de lo que se dice en él” (1992, p. 149). De esa forma, será auto-olvido en cuanto que el verdadero ser del lenguaje se constituye en el mundo en que viven las personas. Ese mundo de las tradiciones y de los prejuicios en que están sumergidas, y que presuponen todo aquello que realizan o dicen, pero que permanece como el umbral inconsciente de nuestro hacer y pensar.

\section{El Hombre como Ser en el Mundo y la Comprensión}

Ese auto-olvido es lo que caracteriza al hombre como un ser-en-elmundo. Ello significa que el mundo no es un objeto que esté allí a disposición del hombre, sino que "es primordialmente para el hombre aquello dentro y en medio de lo que está" (Gadamer, 1993, p. 121). No obstante, también podría decirse que los animales están en el mundo. Sin embargo, aquello que define esta relación del hombre con el mundo es la libertad que posee frente al entorno, libertad que lo hace capaz de apropiarse de las cosas: "elevarse por encima de las coerciones de lo que le sale a uno al encuentro desde el mundo significa tener lenguaje y tener mundo" (Gadamer, 1977, p. 532). 


\section{EL LENGUAJE Y LA COMPRENSIÓN DEL OTRO EN \\ LA HERMENÉUTICA DE GADAMER}

La cita anterior nos servirá para explorar más términos. El libre comportamiento del hombre se explica a partir de la diferencia entre "mundo" y “entorno". Entorno vendrá a ser el medio en el que uno vive, lo que caracterizará a un ser vivo. En cambio, el mundo solo lo posee el hombre, en cuanto que está representado en un lenguaje

Así, 'estar en el mundo' implica un comportamiento libre respecto del mundo. Pero mediante su libertad el hombre no abandona su entorno, sino que adopta un comportamiento distante respecto de él: "Un comportamiento libre y distanciado cuya realización siempre es lingüística” (Gadamer, 1977, p. 533). Ahora bien, Gadamer piensa que esa condición del comportamiento distante que caracteriza el estar en el mundo empuja al hombre a buscar el lenguaje y, por ende, a la comprensión de lo que es el mundo: "comprender, entender (Verstehen) es comprenderse, entenderse (Sich-Verstehen) en el mundo" (1993, p. 121). Así, de la condición humana del estar inmerso en el mundo se desprende que la comprensión involucra necesariamente la comprensión del mundo.

No obstante, el mundo es un espacio compartido con otros. Por eso, el estar en el mundo, o mejor dicho, comprender el mundo significará entenderse unos con otros. Y agrega Gadamer, incluso, que ese entender al otro tiene una “intención moral, no lógica” (1993, p. 123). De esta manera, esta concepción del estar en el mundo contraerá consecuencias para la filosofía práctica, pues dicha comprensión supone una tarea moral en tanto apela a la solidaridad entre los hombres. Así, la comprensión del mundo supondrá una solidaridad o un mutuo entendimiento entre las diversas culturas, que solo se hará posible si los hombres saben aprovechar los recursos del lenguaje.

Ello se encontrará en relación con el fenómeno de la comprensión, pues la condición de esa solidaridad radicará en que los hombres estén dispuestos a escucharse unos a otros, es decir, que sean capaces de dialogar. En relación a ello, Gadamer insistirá en la importancia del escuchar al otro al reflexionar sobre 
la palabra auditorio: "Todos somos auditorio, debemos aprender a escuchar, en uno u otro camino, a luchar siempre contra el ensimismamiento y eliminar $e l$ egoísmo y el afán de imposición de todo impulso intelectual” (Gadamer, 2000, p. 147). ${ }^{1}$ Por esa razón, puede insistirse en que la esencia del lenguaje no radica en su instrumentalización, sino en el diálogo o la conversación:

El lenguaje sólo tiene su verdadero ser en la conversación, en el ejercicio del mutuo entendimiento. (...) El entendimiento como tal no necesita instrumentos en el sentido auténtico de la palabra. Es un proceso vital en el que vive su representación una comunidad de vida. (1977, p. 535)

Visto de esa manera, el diálogo no es una simple herramienta que permita la entendimiento y haga más fácil la convivencia entre las personas. Al contrario, comenta Agustín Domingo, el diálogo ocupará un lugar prioritario en el conjunto de la existencia humana, ya que es "el foco que proporciona luz a la lingüisticidad de la experiencia humana del mundo" (2004, pp. 76-77).

\section{La Dimensión Práctico-Política del Lenguaje}

Por otra parte, el mundo lingüístico no es una barrera que impida acceder al conocimiento del mundo, por el contrario estará abierto a todo aquello que alcanza la percepción, permaneciendo accesible a otros mundos. Esta afirmación tornará problemático, como dirá Gadamer, el concepto de

1 Para un estudio complementario sobre la importancia del escuchar en el quehacer hermenéutico, se puede consultar el artículo de Cecilia Monteagudo (2009b). 


\section{EL LENGUAJE Y LA COMPRENSIÓN DEL OTRO EN \\ LA HERMENÉUTICA DE GADAMER}

mundo en sí, porque la finitud de la experiencia humana mostrará al hombre que siempre se estará en una acepción del mundo, y nunca se alcanzará la acepción 'en sî́ del mundo. De modo que lo propio de cada acepción del mundo es que cada una se mantenga en una relación con la totalidad, aunque solo sea una perspectiva de la totalidad:

Toda acepción del mundo refiere al ser en sí de éste. Él es el todo al que se refiere la experiencia esquematizada lingüísticamente. La multiplicidad de tales acepciones del mundo no significa relativización del mundo. Al contrario, lo que el mundo es no es nada distinto de las acepciones en las que se ofrece. (Gadamer, 1977, p. 536)

Así, Gadamer recuerda que toda acepción del mundo presupone ya lingüisticidad, esto es, una relación intrínseca entre el lenguaje y el mundo que posibilita su comprensión. Pero, además, la parcialidad de cada acepción no es algo que limite la experiencia hermenéutica. Más bien, la perspectiva de cada acepción es fundamental para la experiencia hermenéutica, es incluso condición de posibilidad para comprender otra acepción (Monteagudo, 2009a).

Estas acepciones del mundo representan matizaciones lingüísticas donde cada una de ellas contiene en potencia a las demás matizaciones, porque cada acepción del mundo está potencialmente dispuesta a ampliarse hacia las demás. Dicha ampliación implica la posibilidad de comprender otra visión del mundo, es decir, al otro que interpreta el mundo desde su perspectiva. De esa forma, Gadamer reconocerá la dimensión prácticopolítica de esa ampliación cuando dice: 
La diversidad de las lenguas y la comprensión del mundo es un tema de la máxima actualidad. En el fondo, se trata del tema político por excelencia por el que deberemos responder ante la historia de la humanidad. (1993, p. 111)

En ese sentido, actualmente existen investigaciones acerca del potencial político que hay en las diversas acepciones lingüísticas del mundo. Dicha mediación lingüística radica en el poder para crear vínculos entre diversas comunidades humanas, aunque al mismo tiempo sería el poder para distorsionar los vínculos haciendo de ellos fuentes de destrucción y violencia (Monteagudo, 2009a). ${ }^{2}$ De acuerdo con ello, señalará Cecilia Monteagudo (2005), la concepción gadameriana del lenguaje como acepción del mundo representa claramente un giro hacia la dimensión ético-política de la existencia, en cuanto dicha concepción demanda un cultivo del lenguaje como diálogo. Es por ello que Gadamer considera la diversidad de las lenguas como un tema político, pues desde esta perspectiva dialógica del lenguaje, las diversas comunidades pueden lograr un entendimiento común y establecer vínculos entre ellas que promuevan una convivencia pacífica.

Finalmente, Gadamer descubrirá que esta concepción del lenguaje confiere una especie de 'misión' para la filosofía de nuestro tiempo y en este mundo pluralista: "Este mundo pluralista contiene tareas, que consisten no tanto en la programación y planificación racionalizadoras cuanto en la salvaguardia de los espacios libres de la convivencia humana, incluso por encima de lo extraño" (1993, p. 128). Estos espacios libres no están

2 Con respecto a este estudio, se sugiere consultar el libro de Juan Carlos Godenzzi como referencia de estas investigaciones sobre el potencial político del lenguaje (Godenzzi, 2005). 


\section{EL LENGUAJE Y LA COMPRENSIÓN DEL OTRO EN \\ LA HERMENÉUTICA DE GADAMER}

construidos por determinadas instituciones, ni normas preestablecidas, sino que se fundan simplemente en el diálogo y en el intercambio de ideas. En tal sentido, se entiende que el lenguaje sea el verdadero centro del ser humano, porque representa universalmente el ámbito del consenso siempre mayor (Gadamer, 1992). ${ }^{3}$

Puede decirse, pues, que esta concepción hermenéutica del lenguaje enfrenta a las personas a una praxis vital, una praxis que tiene su punto de partida en la aceptación de la diversidad humana y la pluralidad de las lenguas, y que involucra la escucha y el cuidado del otro como otro. Esta es la consecuencia política más importante de la hermenéutica gadameriana, que no reclama una teoría política, sino una praxis concreta. Una praxis que se define también como una actitud de obediencia, pues se trata de que los hombres obedezcan a "un franco sentimiento de responsabilidad por la humanidad y su destino" (Gadamer, 1993, p. 126).

\section{Conclusiones}

En síntesis, para mostrar la relación intrínseca entre lenguaje y mundo, Gadamer acude a la definición humboldtiana de lenguaje como acepción del mundo, que define la existencia del mundo como determinada por su representación en el lenguaje. En primer lugar, esta relación intrínseca lenguaje-mundo tiene implicancias antropológicas, porque define al hombre como un ser que vive inmerso en el lenguaje. Así entendido, el lenguaje en cuanto tal posee una dimensión práctica, pues como forma de vida tiene la

Asimismo, se recomienda la consulta del artículo de Luis Aguilar en que se destaca también la importancia que confiere Gadamer al lenguaje para la formación humana y la construcción de un mundo más humano (Aguilar, 2003). 
posibilidad de ampliarse hacia otras formas de vida, y por tanto, hace factible la comprensión y la escucha de la alteridad. Y en segundo lugar, como se ha observado, la definición gadameriana de lingüisticidad está en conexión con el concepto filosófico de hombre como ser-en-el-mundo. El término mundo alude al espacio compartido con otros, lo que significa que el hombre en cuanto tal es un ser perteneciente a una historia y una tradición determinada.

Pero, además, esta concepción antropológica sugiere que el hombre en tanto ser-en-el-mundo está ahí para entenderse con los otros que habitan el mismo mundo. De ahí que pueda colegirse que el hombre, por su constitución lingüística y dialógica, esté siempre orientado a la construcción de solidaridades entre las diversas culturas y religiones, mediadas por el lenguaje como diálogo. Dicho de otro modo, la comprensión o el entendimiento del otro mediado de forma lingüística supone la ampliación del horizonte del intérprete, la inclusión de la otra perspectiva y la creación de espacios comunes. En tal sentido, la concepción hermenéutica del lenguaje es un aporte fundamental para pensar nuestra sociedad multicultural por lo que implica la aceptación de la pluralidad y la escucha al otro. De ahí que pueda concluirse que esta concepción hermenéutica del lenguaje involucre una dimensión práctica de gran relevancia para este mundo pluricultural. 


\section{EL LENGUAJE Y LA COMPRENSIÓN DEL OTRO EN \\ LA HERMENÉUTICA DE GADAMER}

\section{Referencias}

Aguilar, L. (2003). Formar en el diálogo, la comprensión y la solidaridad para habitar un mundo tecnificado. Contribuciones de Hans-Georg Gadamer para una formación integral. Analogía filosófica. Revista de filosofía, (1), 67-85.

Domingo, A. (2004). Diálogo y responsabilidad. Claves de la filosofía moral y política de Gadamer. En Acero y Nieves, J. (Ed.), El legado de Gadamer (pp. 63-87). Granada, España: Universidad de Granada.

Gadamer, H. (1977). Verdad y método. Fundamentos para una hermenéutica filosófica. Salamanca, España: Sígueme.

Gadamer, H. (1992). Verdad y método II. Salamanca, España: Sígueme.

Gadamer, H. (1993). Arte y verdad de la palabra. Barcelona, España: Paidós.

Gadamer, H. (1998). El giro hermenéutico. Madrid, España: Cátedra.

Gadamer, H. (2000). La herencia de Europa: ensayos. Barcelona, España: Península.

Gadamer, H. (2001). Antología. Salamanca, España: Sígueme.

Gadamer, H. (2002). Acotaciones hermenéuticas. Madrid, España: Trotta.

Godenzzi, J. (2005). En las redes del lenguaje. Cognición, discurso y sociedad en los Andes. Lima, Perú: Universidad del Pacífico.

Grondin, J. (2003). Introducción a Gadamer. Barcelona, España: Herder. 


\section{JOSÉ LUIS OBREGÓN CABRERA}

Monteagudo, C. (2005). Actitud fenomenológica y actitud hermenéutica en la filosofía de Hans Georg Gadamer. Textos PUCP. Recuperado de http://textos.pucp.edu.pe/pdf/1639.pdf

Monteagudo, C. (2009a). Gadamer y la aplicación de su ontología del lenguaje al fenómeno del Babel contemporáneo. En Cépeda, M. \& Arango, R. (Comps.), Amistad y alteridad: Homenaje a Carlos B. Gutiérrez (pp. 283-292). Bogotá, Colombia: Universidad de los Andes.

Monteagudo, C. (2009b). Escucha y diálogo en el pensamiento de HansGeorg Gadamer. En Monteagudo, C. \&Tubino, F. (Eds.), Hermenéutica en diálogo. Ensayos sobre alteridad, lenguaje e interculturalidad (pp. 5162). Lima, Perú: Fondo Editorial PUCP.

Palmer, R. (2002). ¿Qué es la hermenéutica? Teoría de la interpretación en Schleiermacher, Dilthey, Heidegger y Gadamer. Madrid, España: Arco Libros.

Reyes, J. (2006). ¿Qué papel desempeña el lenguaje en la ontología hermenéutica de Gadamer? Sobre el capítulo 12 de Verdad y método. En Aguilar, M., Entresurcos de Verdad y método (pp. 169 - 194). México D.F., México: UNAM, Facultad de Filosofía y Letras. 